Для цитирования: Миклин Д.Н. Психопатологическая симптоматика (проявления дистресса, тревоги, депрессии и соматизации) у ближайших родственников гематоонкобольных: клинико-динамический аспект. Сибирский вестник психиатрии и наркологии. 2021; 1 (110): 38-43. https://doi.org/10.26617/1810-3111-2021-1(110)$38-43$

\title{
Психопатологическая симптоматика (проявления дистресса, тревоги, депрессии и соматизации) у ближайших родственни- ков гематоонкобольных: клинико-динамический аспект
}

\section{Миклин Д.Н.}

ФГБОУ ВО «Ижевская государственная медицинская академия» Россия, 426034, Июевск, ул. Коммунаров, 281

\section{PEЗЮME}

Цель: изучить психогенные психические расстройства, характеризующиеся проявлениями дистресса, тревоги, депрессии и соматизации у ближайших родственников гематоонкобольных в клинико-динамическом аспекте. Материалы и методы. На базе гематологического отделения БУЗ УР «Первая республиканская клиническая больница» (Ижевск) проведено лонгитюдное исследование у ближайших родственников онкобольных с диагнозом: острый нелимфофобластный лейкоз. В исследовательскую выборку включены мужчины и женщины 18-65 лет. Исследование проводилось в трех точках временного интервала: дебют, 3 и 6 месяцев. Созависимые отношения в семьях изучены по степени родства: по горизонтальной и вертикальной первой линии. Психопатологическая симптоматика пограничного уровня у родственников, вызванная психотравмой, оценивалась с помощью четырехмерного опросника для оценки дистресса, депрессии, тревоги, соматизации (4DSQ - 4ДДТС). Результаты и обсуждение. Наличие у родственника злокачественного заболевания оказывается значительным негативным событием для ближайшего окружения и является фактором нарушения психоэмоционального состояния, что часто приводит к проявлениям депрессии, тревоги, вины и беспомощности в условиях сложившейся кризисной действительности. Постоянно повышенное напряжение в результате стресса в связи с потенциально опасной болезнью предопределяет эмоциональнопсихологическую дезорганизацию, приводящую к дезадаптивным патологическим процессам у родственников онкобольных, ухудшению их психического здоровья. Проведенное исследование позволяет оценить воздействие психогенного фактора в виде злокачественного заболевания у члена семьи на развитие и динамику психопатологической симптоматики у близких родственников. В исследуемой выборке ближайших родственников выявлена высокая встречаемость основных симптомокомплексов: дистресс $(\mathrm{n}=33,35,8 \%)$, соматизация $(\mathrm{n}=18,19 \%)$, депрессия $(\mathrm{n}=18,19,5 \%)$. Установлено, что благодаря внешним и внутренним ресурсам личности адаптивный процесс способствует восстановлению психического состояния родственников к 6-му месяцу лечения. Наиболее интенсивное восстановление происходит в первые 3 месяца, что свидетельствует об эффективности функционирования психологических защит для усиления компенсаторных антистрессовых механизмов высшей нервной деятельности в среднесрочной перспективе и необходимости тактики раннего вмешательства.

Ключевые слова: психоонкология, онкобольные, родственники онкобольных, дистресс, депрессия, тревога, соматизация, психиатричесчкая и психолого-психотерапевтическая помощь, психическое здоровье.

\section{ВВЕДЕНИЕ}

Согласно результатам, представленным в зарубежных и отечественных работах, в последние годы наблюдается значительный рост распространенности психопатологической симптоматики в виде проявлений депрессии, тревоги, дистресса и соматизации $[1,2,3]$. Особую роль в этом авторы отводят онкологическим заболеваниям [4]. Стрессовые ситуации на этапе реакции в ответ на сообщение о подтвержденном диагнозе онкозаболевания испытывают не только сами больные, но и их окружение - близкие родные. Наличие у родственника злокачественного забо- левания оказывается настолько значительным отрицательным событием для ближайшего окружения, что часто приводит к состоянию депрессии, тревоги, вины, беспомощности к сложившейся действительности $[5,6]$. Родные переживают в ответ на стрессовую ситуацию палитру эмоций: желание помочь и ощущение безысходности, боль, страх, надежду и безысходность одновременно, идеи виновности и самообвинения [7]. Сверхнегативность ситуации приводит к эмоциональной и психологической дезорганизации, 
дезадаптивным процессам близких, ухудшению их психического здоровья [8, 9, 10].

Актуальность данной проблемы заключается ещё и в том, что рост онкологической патологии приводит к повышению случаев психогений у созависимого контингента ближайшего окружения [11]. Анализируя публикуемую литературу по психолого-психиатрической проблематике онкологии, становится очевидной актуальность и приоритетность тем, посвященных психическим и психосоматическим расстройствам у онкобольных. В то же время отечественные публикации по изучению психогенных психических расстройств в виде дистресса, тревоги, депрессии и соматизации у ближайших родственников онкогематологических больных, встречаются в литературе достаточно редко $[7,8,12]$.

\section{ЦЕЛЬ РАБОТЫ}

Изучение психопатологической симптоматики у ближайших родственников, характеризующейся проявлениями дистресса, тревоги, депрессии и соматизации с позиций клинико-динамического наблюдения, вызванной психогенным фактором гематологического онкозаболевания у члена их семьи.

\section{МАТЕРИАЛЫ И МЕТОДЫ}

Работа выполнялась на базе гематологического отделении БУЗ Удмуртской республики «Первая республиканская клиническая больница Минздрава Удмуртской Республики». Проведено открытое выборочное клиническое проспективное лонгитюдное исследование ближайших родственников $(\mathrm{n}=92)$ онкогематологических больных (n=92) с верифицированным диагнозом по МКБ-10: острый миелоидный (нелимфобластный) лейкоз (С92.0). В исследовательскую выборку включены лица мужского и женского пола в возрасте от 18 до 65 лет. Созависимые отношения в семьях изучались по степени родства: по горизонтальной и вертикальной первой линии. Исследование проводилось в трех точках временного интервала: дебют, 3 и 6 месяцев. В первой выборке (больных) доля женщин была меньше $(\mathrm{n}=40$, $43,5 \%)$, чем мужчин $(n=52,56,5 \%)$, их средний возраст составил 43,09 $\pm 14,79$ года, медиана (Me) - $44[30,25 ; 55]$. Во второй выборке (родственников) женщин оказалось больше $(n=59,64,1 \%)$, чем мужчин $(\mathrm{n}=33,35,9 \%), \quad$ средний возраст -

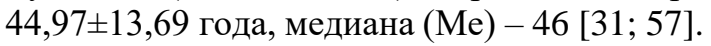

В период исследования изначальная численность $(\mathrm{n}=92)$ рассматриваемой выборки прогрессивно снижалась по причине летальности больных от основного заболевания - острый нелимфобластный лейкоз. В 3-месячный временной период в группе родственников $(\mathrm{n}=88)$ участвовали 59 женщин и 29 мужчин. К концу 6-месячного периода выборка ближайшего окружения $(\mathrm{n}=70)$ сократилась ещё больше (49 женщин и 21 мужчина).

Опрос и беседа с больными осуществлялась в палате интенсивной терапии гематологического отделения, общение с родственниками больных (информация о состоянии его здоровья, методах различных обследований, вариантах лечения, прогнозе заболевания) - на приеме у врача в момент постановки диагноза, через 3 и 6 месяцев.

Для решения поставленных целей и задач оценивали эмоциональное и психовегетативное напряжение, скрытую тревогу и депрессию, соматовегетативную недостаточность и ранние симптомы соматизированных психических расстройств на основе четырехмерного голландского опросника для оценки дистресса, депрессии, тревоги и соматизации, адаптированного к российской популяции (4ДДТС) [13, 14, 15].

Статистическая обработка полученных результатов осуществлялась с применением программы SPSS 22. Методами математической статистики осуществлен расчет средних значений (M), стандартного отклонения $( \pm \sigma)$, медианы $(\mathrm{Me})$, нижнего и верхнего квартилей [25;75].

\section{РЕЗУЛЬТАТЫ И ОБСУЖДЕНИЕ}

В результате изучения и анализа анамнестических сведений выявлены основные социальнодемографические характеристики ближайших родственников гематоонкобольных. Возраст родственников приходился на диапазон от 18 до 65 ле. Средний возраст родственников в дебюте заболевания имел примерно одинаковые показате-

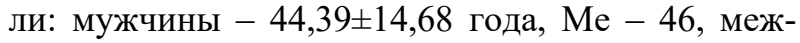
квартильный размах [29;58], женщины $45,3 \pm 13,22$ года, Ме - 46 [31;56]. В течение 3 месяцев существенных изменений по величине среднего возраста у респондентов не выявлено. Через 6 месяцев средний возраст родственников незначительно изменился: мужчины $42,75 \pm 14,09$ года, Ме - 43 [28,5;56], женщины $45,6 \pm 12,77$ года, Me - 46 [32,5;56,25].

На момент первоначального исследования родственников гематоонкобольных $(n=92)$ менее пятой части $(\mathrm{n}=21,22 \%)$ являлись пенсионерами, преобладающее большинство $(\mathrm{n}=71,78 \%)$ были работниками различных сфер деятельности. Высшее образование выявлено незначительно реже, чем среднее специальное образование: $\mathrm{n}=38$ (41\%) против $\mathrm{n}=54$ (59\%). Причем женщин с высшим образованием зарегистрировано больше, чем мужчин (63,2\% против $36,8 \%)$. Среди родственников большинство $(\mathrm{n}=71)$ состояли в законном браке, из них доля состоящих в зарегистрированном браке женщин $(\mathrm{n}=44,62 \%)$ была выше, чем мужчин $(\mathrm{n}=27,38 \%)$. Из родственников гематоонкобольных одного ребенка имели 27 
респондентов (29,3\%), двух детей - 39 (42,4\%), трех - 11 (12,0\%), не имели детей - 15 (16,3\%).

В выборке родственников гематоонкобольных $(\mathrm{n}=92)$ преобладали представители русской национальности $(\mathrm{n}=65,70,2 \%)$ по сравнению с лицами удмуртской $(\mathrm{n}=17,18 \%)$ и татарской $(\mathrm{n}=10$, $10,8 \%)$ национальностей. Более трети $(37 \%)$ исследованных родственников имели патологию желудочно-кишечного тракта, у 23\% респонден- тов выявлены заболевания сердечно-сосудистой системы, причем родственники отличались преобладанием парасимпатической регуляции вегетативной нервной системы $(\mathrm{n}=72,78,3 \%)$. Частота поражения других систем организма была представлена единичными случаями.

У родственников гематоонкобольных были определены средние значения баллов по четырем шкалам опросника 4ДДТС (табл. 1).

Т а б л и ц а 1 . Сравнительная характеристика средних значений показателей у родственников гематоонкобольных по опроснику 4ДДТС в дебюте заболевания, через 3 и 6 месяцев

\begin{tabular}{|c|c|c|c|c|c|c|c|c|c|}
\hline \multirow{3}{*}{$\begin{array}{c}\text { Шкала опросника } \\
\text { 4ДДТС }\end{array}$} & \multicolumn{9}{|c|}{ Средние значения показателей по опроснику 4ДДТС } \\
\hline & \multicolumn{3}{|c|}{ Дебют онкозаболевания } & \multicolumn{3}{|c|}{ Через 3 месяца } & \multicolumn{3}{|c|}{ Через 6 месяцев } \\
\hline & $\mathrm{M} \pm \sigma$ & $\mathrm{Me}$ & {$[25 ; 75]$} & $\mathrm{M} \pm \sigma$ & $\mathrm{Me}$ & {$[25 ; 75]$} & $\mathrm{M} \pm \sigma$ & $\mathrm{Me}$ & {$[25 ; 75]$} \\
\hline Соматизация & $7,7 \pm 6,02$ & 6,0 & {$[4 ; 10]$} & $6,97 \pm 5,63$ & 5,5 & {$[3 ; 10]$} & $6,97 \pm 5,97$ & 6,0 & {$[2 ; 10]$} \\
\hline Дистресс & $9,94 \pm 6,55$ & 9,0 & {$[5,25 ; 13]$} & $7,26 \pm 6,42$ & 5,5 & {$[2 ; 11]$} & $7,23 \pm 5,9$ & 6,0 & {$[2,5 ; 11]$} \\
\hline Тревога & $3,41 \pm 3,6$ & 2,0 & {$[0,25 ; 6]$} & $2,39 \pm 3,47$ & 1,0 & {$[0 ; 4]$} & $2,44 \pm 3,32$ & 1,0 & {$[0 ; 5]$} \\
\hline Депрессия & $0,89 \pm 1,55$ & 0 & {$[0 ; 1]$} & $1,06 \pm 2,17$ & 0 & {$[0 ; 1]$} & $0,59 \pm 1,27$ & 0 & {$[0 ; 1]$} \\
\hline
\end{tabular}

П р и м е ч а н и е: М - среднее значение, б - среднеквадратичное отклонение, Ме - медиана, межквартильный размах $-[25 ; 75]$.

Выявлено, что средние значения показателей у родственников гематоонкобольных к 6-му месяцу течения болезни неуклонно снижаются. Причем наибольшая выраженность происходящего снижения наблюдается по показателю «де-

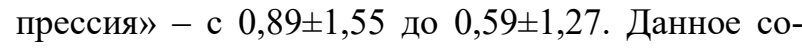
стояние может рассматриваться как адаптивный стимулирующий процесс, исходя из имеющихся личностных ресурсов (внешних или внутренних), когда в ситуации болезни члена семьи и угрозе безопасности всей семейной системы наблюдается осознание происходящего, принятие реальности, вырабатываются защитные механизмы, происходит адаптация к формирующимся условиям.

Из общей совокупности исследуемых родственников онкобольных нами были выделены респонденты с высоким уровнем показателей анкеты 4ДДТС. Данные представлены в таблице 2.

Т а бл и ц а 2. Сравнительная характеристика психовегетативных показателей с высокой и средней степенью выраженности у родственников гематоонкобольных в дебюте заболевания, через 3 и 6 месяцев

\begin{tabular}{|l|c|c|c|}
\hline \multirow{2}{*}{$\begin{array}{c}\text { Шкала опросника } \\
\text { 4ДДТС }\end{array}$} & \multicolumn{2}{|c|}{ Показатели с высокой и средней степенью выраженности у родственников гематоонкобольных } \\
\cline { 1 - 4 } Соматизация & $\mathrm{n}=18(19,5 \%)$ & $\mathrm{Через} \mathrm{3} \mathrm{месяца}$ & Через 6 месяцев \\
\hline Дистресс & $\mathrm{n}=33(35,8 \%)$ & $\mathrm{n}=20(22,7 \%)$ & $\mathrm{n}=10(14,3 \%)$ \\
\hline Тревога & $\mathrm{n}=6(6,5 \%)$ & $\mathrm{n}=6(6,8 \%)$ & $\mathrm{n}=5(7,1 \%)$ \\
\hline Депрессия & $\mathrm{n}=18(19,5 \%)$ & $\mathrm{n}=16(18 \%)$ & $\mathrm{n}=7(10 \%)$ \\
\hline
\end{tabular}

П р и м е ч а н и е: $\mathrm{n}$ - абсолютный показатель, \% - относительный показатель.

Анализируя динамику изменения психовегетативных показателей у родственников гематоонкобольных в трех изучаемых временных срезах, можно констатировать, что в дебюте заболевания родственники гематоонкобольных находятся в состоянии дезорганизации, что не позволяет осмыслить проблему в целом, сделать выбор оптимальной стратегии действий, принять логически-последовательные решения. На данном этапе на родственников оказывает влияние совокупность внешних и внутренних феноменов; взаимодействуя друг с другом, они подчиняются в ситуации нарастающего кризиса каким-то другим правилам и законам, которые не могут быть объяснены с позиций рациональности и математической логики [12]. Согласно исследованиям, про- веденным в Центре сотрудничества ВОЗ по паллиативному лечению (Оксфорд) [16], эмоциональные переживания развиваются в изменившихся условиях жизни и достигают максимального напряжения в ситуации постановки онкологического диагноза, что совпадает с результатами нашего исследования.

Понимание кризисной и драматичной ситуации угрозы жизни близкого человека, в которой родственники гематоонкобольных оказались роковым образом - сложный, многогранный и многофакторный процесс. На психоэмоциональную составляющую родственников оказывает влияние своеобразие семейной жизнедеятельности, личностно-психологическая устойчивость членов семьи и индивидуальная резистентность к стрес- 
су, наличие прошлого опыта преодоления трудных жизненных ситуаций [17].

Система семейного функционирования в условиях фрустрирующего воздействия трансформируется одновременно с процессом адаптации ближайшего окружения с выработкой нового уровня поведения и деятельности к 3-му месяцу течения заболевания. Родственники пытаются найти запасной источник ресурсов в самой окружающей жизни. Для одних это социальная поддержка или обращение к религии, для других забота и родственная поддержка близкого человека начинает приобретать особый смысл в новой жизни, имея ввиду способность помочь тем, кого любили [12]. В этот промежуточный период происходит наиболее интенсивное восстановление, что свидетельствует о значении психологических защит личности в системе компенсаторных механизмов высшей нервной деятельности в среднесрочной перспективе и необходимости применения тактики раннего вмешательства.

\section{ЗАКЛЮЧЕНИЕ}

В результате проведенного исследования психогенных психических расстройств, характеризующихся проявлениями дистресса, тревоги, депрессии и соматизации у ближайших родственников гематоонкобольных в клинико-динамическом аспекте, можно сформулировать основные выводы.

Изучение выборки родственников онкогематобольных с диагнозом: острый нелимфобластный лейкоз в структуре психогенных психических расстройств показало частую встречаемость следующих проявлений на момент дебюта заболевания: дистресс $(\mathrm{n}=33,35,8 \%)$, соматизация $(\mathrm{n}=18,19,5 \%)$, депрессия $(\mathrm{n}=18,19,5 \%)$, тревога $(\mathrm{n}=6,6,5 \%)$.

Адаптивный процесс с приспособлением к изменениям к микросреде (больной член семьи) благодаря внешним и внутренним ресурсам личности способствует восстановлению психической устойчивости родственников к 6-му месяцу лечения заболевания, что подтверждается снижением изучаемых показателей: дистресс (n=16, 22,8\%), соматизация $(\mathrm{n}=10,14,3 \%)$; депрессия $(\mathrm{n}=7,10,0 \%)$, тревога $(\mathrm{n}=5,7,1 \%)$.

Более интенсивное восстановление происходит в первые 3 месяца, что отражается в более форсированном снижении изучаемых показателей в трехэтапном наблюдении: дистресс $(22,8 \%)$, соматизация (12,5\%), депрессия $(18,0 \%)$, тревога $(6,8 \%)$ и подтверждает высокую значимость психологических защит личности в системе компенсаторных механизмов высшей нервной деятельности в среднесрочной перспективе и необходимости выбора тактики раннего вмешательства.

Для коррекции уровня тревожности при раннем вмешательстве необходимо назначение анксиолитиков, бензодиазепинов, препаратов ГАМК, снижающих уровень деструктивной тре- воги. С целью снижения проявлений депрессии назначаются антидепрессанты с анксиолитическим и рединамизирующим действием на срок не менее 2 месяцев. При повышенном уровне дистресса и нервного напряжения эффективно воздействуют дебрифинг, позитивная и рациональная психотерапия с опорой на продуктивный прошлый опыт совладания с дистрессом. Проявления соматизации нивелируют методами катарсической терапии, терапии первичным криком, динамической медитации, кинезиотерапии.

\section{КОНФЛИКТ ИНТЕРЕСОВ}

Автор заявляет об отсутствии возможных конфликтов интересов в связи с публикацией материалов данной статьи.

\section{ИСТОЧНИК ФИНАНСИРОВАНИЯ}

Исследование не имело спонсорской или иной финансовой поддержки.

\section{СООТВЕТСТВИЕ ПРИНЦИПАМ ЭТИКИ}

Настоящее исследование выполнено в соответствии с руководством ICN по доброкачественной клинической практике, правилами GCP (Good Clinical Practice) и Хельсинской декларацией BMA по проведению биометрических исследований на людях. Исследование проводилось на основе информированного согласия по п. 4.6.1. приказа № 163 (ОСТ 9/500.14.001 - 2002) МЗ РФ.

\section{ЛИТЕРАТУРА/REFERENCES}

1. Говорин Н.В., Бодагова Е. А. Психическое здоровье и качество жизни врачей. Томск, Чита: Изд-во Иван Федоров, 2013. 124 c. Govorin NV, Bodagova EA. Mental health and quality of life of doctors. Tomsk, Chita: Publishing House "Ivan Fedorov", 2013:122 (in Russian).

2. Онкопсихология. Руководство для врачейонкологов и медицинских психологов / ред. А.М. Беляев, М.В. Рогачев, Т.Ю. Семиглазова, В.А.Чулкова. СПБ. : АННМИ «Вопросы онкологии», 2017. 350 c. Onco-psychology. Handbook for oncologists and clinical psychologists. AM Belyaev, MV Rogachev, TYu Semiglazova, VA Chulkova, eds. St. Petersburg: Autonomous non-for-profit scientific and medical organization "Oncology Issues", 2017:350 (in Russian).

3. Akechi T, Akizuki N, Okamura M, Shimizu K, Oba A, Ito T, Yoshikawa E, Nakano T, Inagaki M, Uchitomi Y. Psychological distress experienced by families of cancer patients: preliminary findings from psychiatric consultation of a Cancer Center Hospital. Jpn J Clin Oncol. 2006 May;36(5):32932. doi: 10.1093/jjco/hyl029

4. Иванов С.В. Депрессивные расстройства в онкологии (обзор). Журнал неврологии и психиатрии им. С.С. Корсакова. 2012. Т. 112(11). С. 104109. Ivanov SV. Depressive disorders in oncology (review). S.S. Korsakov Journal of Neurology and Psychiatry. 2012;112(11):104-109 (in Russian).

5. Реверчук И.В. Теоретические и методологические противоречия становления биопсихосоци- 
альной парадигмы. Вестник психиатрии и психологии Чувашии. 2008. № 4. С. 109-123. Reverchuk IV. Theoretical and methodological contradictions in the formation of the biopsychosocial paradigm. Bulletin of Psychiatry and Psychology of Chuvashia. 2008;4:109-123 (in Russian).

6. Костина Н.И. Психологическая помощь онкобольным и родственникам на стационарном этапе. Злокачественные опухоли. 2016. № 451 (21). C. 161-162. Kostina NI. Psychological support to cancer patients and relatives at the inpatient stage. Malignant Neoplasms. 2016;451(21): 161162 (in Russian).

7. Гнездилов А.В. Психические изменения у онкологических больных. Практическая онкология. 2001. T. 2, № 1 (05). C. 5-13. Gnezdilov AV. Mental changes in cancer patients. Practical Oncology. 2001;2,No.1(05):5-13 (in Russian).

8. Дейнека Н.В., Курбатова Е.Г. Психологическая характеристика адаптации к болезни при лейкозах. Наука вчера, сегодня, завтра: сборник статей по материалам XLI междунар. научно-практ. конф. Часть I. Новосибирск : Изд-во СибАК. 2016. T. 12 (34). C. 114-118. Deineka NV, Kurbatova EG. Psychological characteristics of adaptation to disease in leukemia. Science yesterday, today, tomorrow: a collection of articles based on the materials of the XLI international scientific and practical conference. Part I. Novosibirsk : Publishing House SibAK. 2016;12(34):114-118 (in Russian).

9. Реверчук И.В., Главатских М.М., Миклин Д.Н. Применение психосемантических методов в диагностике психоэмоционального статуса ближайших родственников онкобольных. Электронный научно-образовательный вестник «Здоровье и образование в XXI веке». 2018. T. 20, № 5. C. 52-57. Reverchuk IV, Glavatskikh MM, Miklin DN. The use of psychosomatic techniques in the diagnosis of mental and emotional status of close relatives of cancer patients. Electronic scientific and educational bulletin "Health and Education in the XXI Century". 2018;20(5):52-57 (in Russian).

10. Реверчук И.В., Главатских М.М., Миклин Д.Н. Клинические особенности стресс-совладающего поведения и качества жизни у женщин - родственниц онкологических больных. Медицинская сестра. 2019. T. 21, 3 1. C. 18-21. Reverchuk IV, Glavatskikh MM, Miklin DN. Clinical features of stress-coping behavior and quality of life in female relatives of cancer patient. Nurse. 2019; 21 (1): 18-21 (in Russian). DOI: 10.29296/25879979-2019-01-05

11. Wittenberg E, Prosser LA. Disutility of illness for caregivers and families: a systematic review of the literature. Pharmacoeconomics. 2013 Jun; 31(6):489-500. doi: 10.1007/s40273-013-0040-y
12. Белинская Е.П., Сангова С. Болезнь как трудная жизненная ситуация: особенности стратегий совладания родственников тяжелобольных [Электронный ресурс]. Психологические исследования. 2015. Т. 8, № 42. С. 8. Belinskaya EP, Sangova $\mathrm{S}$. The illness as a difficult life situation: characteristics of coping strategies of seriously ill patients' relatives [Electronic resource]. Psychological Research. 2015;8(42):8. URL: http://psystudy.ru (дата обращения: 08.11.2020) (in Russian).

13. Смулевич А.Б., Яхно Н.Н., Терлуин Б., Захарова Е.К., Рейхард Д.В., Андрюшенко А.В., Парфенов В.А., Замеград М.В., Арнаутов В.С., Романов Д.В. Четырехмерный опросник для оценки дистресса, депрессии, тревоги и соматизации (4ДДТС) при вегетативных психосоматических расстройствах пограничного уровня. Журнал неврологии и психиатрии им. С.С. Корсакова. 2014; T. 114, № 11. C. 67-73. Smulevich AB, Yakhno NN, Terluin B, Zakharova EK, Reikhard DV, Andryushenko AV, Parfenov VA, Zamegrad MV, Arnautov VS, Romanov DV. Fourdimensional questionnaire for the assessment of distress, depression, anxiety and somatization (4DDTS) in autonomic psychosomatic disorders of the borderline level. S.S. Korsakov Journal of Neurology and Psychiatry. 2014;114(11):67-73 (in Russian).

14. Czachowski S, Terluin B, Izdebski A, Izdebski P. Evaluating the cross-cultural validity of the Polish version of the Four-Dimensional Symptom Questionnaire (4DSQ) using differential item functioning (DIF) analysis. Fam Pract. 2012 Oct;29(5):60915. doi: $10.1093 /$ fampra/cms016

15. Tebbe BB, Terluin B, Koelewijn JM. Assessing psychological health in midwifery practice: a validation study of the Four-Dimensional Symptom Questionnaire (4DSQ), a Dutch primary care instrument. Midwifery. 2013 Jun;29(6):608-15. doi: 10.1016/j.midw.2012.05.004

16. Твайкросс Р.Дж., Фрамптон Д. Введение в паллиативное лечение. Лекции. Оксфорд, 1992. 35 c. Twycross RJ, Frampton D. Introduction to palliative care. Lectures. Oxford, 1992:35 (in Russian) http://www.medpsy.ru/society/society001.php

17. Куфтяк Е.В. Жизнеспособность семьи: теория и практика Медицинская психология в России: электронный научный журнал. 2014. № 5 (28) [Электронный pecypc]. Kuftyak EV. Family vitality: theory and practice Medical psychology in Russia: Electronic Scientific Journal. 2014;5(28) [Electronic resource]. URL: http://mprj.ru (дата обращения: 08.11.2020) (in Russian).

Поступила в редакцию 17.11.2020 Утверждена к печати 03.03.2021 
Миклин Дмитрий Николаевич, аспирант кафедры педагогики, психологии и психосоматической медицины, «Ижевская государственная медицинская академия». ORCID iD 0000-0002-6614-124X.

Миклин Дмитрий Николаевич, miklin.miklin@yandex.ru

UDC 616.89.4:616.891.6:616.8-008.64:615.155-056.24:614.253.89

For citation: Miklin D.N. Psychopathological symptoms (manifestations of distress, anxiety, depression and somatization) in the closest relatives of patients with blood cancer: clinical and dynamic aspect. Siberian Herald of Psychiatry and Addiction Psychiatry. 2021; 1 (110): 38-43. https://doi.org/10.26617/1810-3111-2021-1(110)-38-43

\title{
Psychopathological symptoms (manifestations of distress, anxiety, depression and somatization) in the closest relatives of patients with blood cancer: clinical-dynamic aspect
}

\section{Miklin D.N.}

Izhevsk State Medical Academy

Kommunarov Street 281, 426034, Izhevsk, Russian Federation

\begin{abstract}
Objective: to study psychogenic mental disorders characterized by manifestations of distress, anxiety, depression and somatization in the closest relatives of patients with blood cancer in the clinical-dynamic aspect. Materials and Methods. Based on the Blood Cancer Department of the First Republican Clinical Hospital (Izhevsk), the longitudinal study was carried out in the closest relatives of cancer patients diagnosed with acute non-lymphoblastic leukemia. The study sample included men and women aged 18-65 years. The study was carried out at three points in the time interval: debut, 3 and 6 months. Codependent relationships in families were studied according to the degree of relationship: along the horizontal and vertical first line. Psychopathological symptoms of the borderline level caused by trauma in relatives were assessed using a four-dimensional questionnaire to evaluate distress, depression, anxiety, and somatization (4DSQ). Results and Discussion. The presence of a malignant disease in a relative appeared to be a significant negative event for the nearest and was a factor in the disturbance of the psychoemotional state, which often lead to manifestations of depression, anxiety, guilt and helplessness in the current crisis reality. Increasing tension as a result of stress in connection with the potentially dangerous illness predetermined emotional and psychological disorganization, leading to maladaptive pathological processes in relatives of cancer patients, deterioration of their mental health. The study allowed us to assess the impact of a psychogenic factor in the form of a malignant disease in a family member on the development and dynamics of psychopathological symptoms in close relatives. In the studied sample of close relatives, the high incidence of basic symptom complexes was revealed: distress $(n=33,35.8 \%)$, somatization $(n=18,19 \%)$; depression $(n=18,19.5 \%)$. It was found that due to the external and internal resources of the personality, the adaptive process contributed to the recovery of the mental state of relatives by month 6 th of the treatment. The most intensive recovery occurred in the first three months, which indicated the effectiveness of the functioning of psychological defenses to enhance the compensatory anti-stress mechanisms of higher nervous activity in the medium-term perspective and the need for early intervention tactics.
\end{abstract}

Keywords: psycho-oncology, cancer patients, relatives of cancer patients, distress, depression, anxiety, somatization, mental health services, mental health.

Received November 17.2020

Accepted March 03.2021

Miklin Dmitry N., post-graduate student of the Department of Pedagogy, Psychology and Psychosomatic Medicine, Izhevsk State Medical Academy. ORCID iD 0000-0002-6614-124X.

Miklin Dmitry N., miklin.miklin@yandex.ru 